\title{
Riboflavin transporter deficiency
}

\author{
INSERM
}

\section{Source}

INSERM. (1999). Orphanet: an online rare disease and orphan drug data base. Riboflavin transporter deficiency. ORPHA:97229

Riboflavin transporter deficiency (RTD) is a progressive motor neuron disorder characterized by respiratory insufficiency, sensorineural deafness and progressive pontobulbar palsy. 